\title{
Targeting PD-L1 After Adjuvant Radiation in Subtotally Resected Primary Pineal Melanoma: A Case Report and Literature Review
}

\author{
Justin Famoso, MDa; Gerald Lemole, MD ${ }^{\mathrm{b}}$; Srinath Sundararajan, $\mathrm{MD}^{\mathrm{c}}$; and Baldassarre Stea, MD, $\mathrm{PhD}^{\mathrm{a}}$
}

\section{ABSTRACT}

Primary melanoma of the pineal gland is a rare disease entity with an overall poor prognosis. Limited data exist to appropriately guide treatment decisions. Historical case reports have showed some success using a combination of surgical resection, radiotherapy, and chemotherapy, but long-term survival has been exceedingly rare. This report presents a female patient with a primary pineal melanoma who underwent subtotal resection followed by adjuvant focal radiation to the residual tumor. Immunohistochemistry identified a strong positivity for PD-L1 (70\%). After radiation, systemic therapy with pembrolizumab was initiated with the plan to treat until progression. She has now completed 33 cycles of pembrolizumab without interruptions, complications, or disease progression. At the time of writing, the patient has had an excellent clinical outcome, with a durable near-complete response of $>138$ weeks. To our knowledge, this is the first patient with a pineal melanoma to be managed by targeting PD-L1. Furthermore, she has achieved the second longest overall survival and the longest progression-free survival reported in the literature.

J Natl Compr Canc Netw 2019;17(10):1148-1153 doi: $10.6004 /$ jnccn.2019.7336

\footnotetext{
${ }^{a}$ Department of Radiation Oncology, ${ }^{b}$ Division of Neurosurgery, and ${ }^{c}$ Division
} of Medical Oncology, The University of Arizona, Tucson, Arizona.
Primary malignant melanoma of the pineal gland is a rare disease entity, with only 24 cases reported in the literature to date. ${ }^{1}$ Given the rarity of this malignancy, management is primarily based on case reports and literature reviews. We present the first reported case of a patient with a subtotally resected pineal melanoma achieving an excellent clinical outcome using a combination of adjuvant radiotherapy (RT) and pembrolizumab, and provide a thorough review of the literature and discussion of current management strategies.

\section{Case Report}

A 75-year-old white woman with a past medical history of hypertension and hypercholesterolemia and a family history of male breast cancer in a paternal uncle and renal cell carcinoma in a son presented to the emergency department with a 10-day history of progressively worsening altered mental status, confusion, and gait disturbance. She complained of vertical gaze paresis and diplopia consistent with Parinaud syndrome. In addition, she complained of increasing difficulty performing tasks requiring fine coordination, eyestrain headaches, worsening gait imbalance, and loss of taste sensation. On physical examination, her vital signs were within normal limits; she was alert and oriented to person and place but exhibited disorientation to time. Her gait was wide-based and slow, strength and sensation were intact in bilateral lower extremities, and she exhibited vertical gaze palsy with downward gaze preserved. All other cranial nerves were intact. No pronator drift was noted, and coordination was appropriate. Results of basic laboratory workup were normal, including blood count with differential, complete metabolic panel, and urinalysis with culture.

The patient underwent a brain CT without contrast, which revealed a poorly defined hyperdense mass in the pineal region associated with moderate obstructive hydrocephalus. Follow-up MRI revealed a homogenously enhancing pineal lesion on T1-wighted images, inseparable from the tectum, that measured 3.4 (anteroposterior) 
$\times 2.6$ (mediolateral) $\times 2.4 \mathrm{~cm}$ (craniocaudal) (Figure 1A) A local mass effect was noted on the tectum and anterior aspect of the brain stem extending into the cerebral aqueduct, with associated perilesional vasogenic edema.

The patient underwent urgent image-guided suboccipital craniotomy for pineal tumor resection using a supracerebellar infratentorial approach. Intraoperative findings revealed a hyperemic-appearing lesion situated in the pineal region with encasement of the precentral cerebellar vein and was severely adherent to portions of the midbrain and brainstem. Given these findings, the tumor was only amenable to partial resection, with residual tumor remaining postoperatively measuring $2.9 \times 2.6 \times 2.4 \mathrm{~cm}$ on MRI (Figure 1B).

Gross pathology revealed a dark red-brown friable mass. Microscopic evaluation showed nests of pleomorphic cells with hyperchromatic nuclei and melanotic pigment. Immunohistochemical staining was positive for S100, Melan-A, and HMB45 and negative for synaptophysin, neurofilament, pankeratin, and cytokeratin. Molecular profiling (MI Profile report, Caris Life Sciences) was performed on the tissue sample and revealed positive immunohistochemistry results $(2+)$ for PD-L1, $70 \%$.
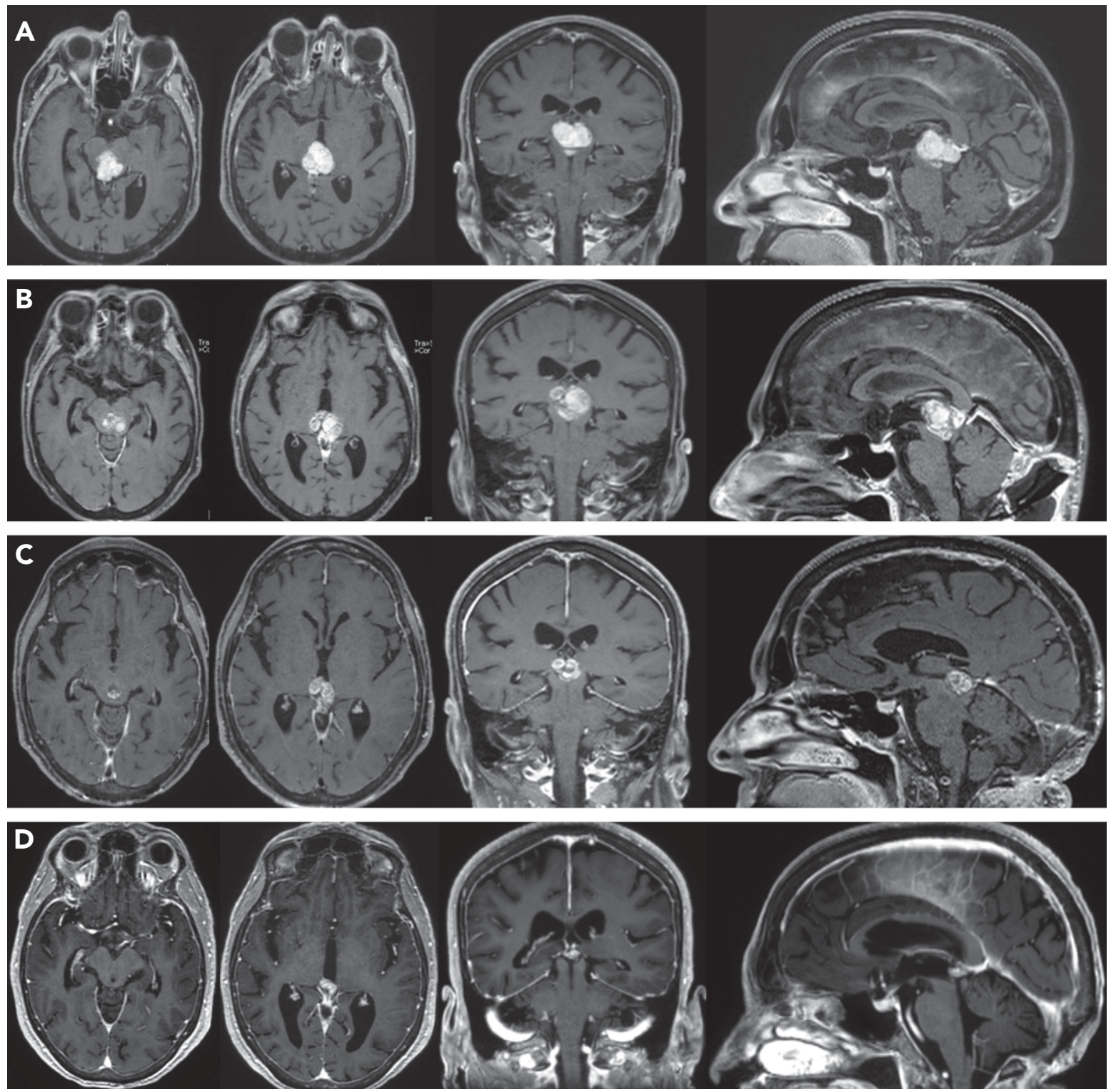

Figure 1. MRI T1 sequence with contrast (A) at presentation, (B) after resection, (C) after radiation, and (D) after 32 cycles of pembrolizumab showing near-complete resolution of the pineal mass.

Each horizontal panel represents a single study with the inferior axial, superior axial, coronal, and sagittal views arranged from left to right. 
In addition, a pathogenic somatic mutation was identified in BAPl on exon 11.

The patient underwent systemic staging, including MRI total spine, total-body PET/CT, and complete skin survey by a dermatologist. All evaluations revealed no evidence of a primary extracranial or metastatic melanomatous lesion. After this workup, the patient was considered to have a primary malignant melanoma of the pineal gland.

Adjuvant RT was initiated 1 month after craniotomy. She received stereotactic intensity-modulated RT to the postoperative bed and residual gross tumor (Figure 2) to a prescribed dose of $54 \mathrm{~Gy}$, delivered in 30 daily fractions of $1.8 \mathrm{~Gy}$. An unplanned interruption in RT occurred for 9 days between fractions 9 and 10 due to epidural abscess with wound dehiscence, which was managed successfully with irrigation and debridement of the occipital wound and intravenous vancomycin and cefepime. She completed her remaining course of RT without issue. MRI performed after completion revealed an interval decrease in size of the residual postoperative enhancing lesion to $2.5 \times 1.9 \times 1.5 \mathrm{~cm}$ (Figure $1 \mathrm{C}$ ).

On completion of RT, the patient was evaluated for systemic therapy options; after approximately 4 weeks, she started a course of pembrolizumab $(2 \mathrm{mg} / \mathrm{kg}$ intravenously, every 3 weeks) because her tumor was significantly PD-L1-positive. Follow-up MRI 2 months after initiating immunotherapy revealed continued response to therapy and interval decrease in size of the pineal mass to $1.6 \times 1.6 \times 1.0 \mathrm{~cm}$.

At the time of writing, the patient has completed 33 cycles of pembrolizumab without interruptions or complications. Her most recent brain MRI continued to show interval decreases in size, measuring $1.3 \times 1.1 \times 0.4 \mathrm{~cm}$ (Figure 1D). She is 138 weeks ( $>32$ months) from initial presentation and 110 weeks from completion of RT ( $>27$ months) without evidence of recurrent or progressive disease.
Clinically, the patient has had a good outcome. Her previously noted altered mental status, headaches, and fine coordination normalized shortly after surgery. Her gait instability showed gradual improvement over the course of RT and continued to improve while on immunotherapy. At the time of writing, she is able to ambulate with assistance from a wheeled-walker. The previously noted vertical gaze palsy and diplopia have been gradually improving in the past few years. Unfortunately, these issues persist, albeit to a lesser extent than at initial presentation. Although she has had no grade $\geq 2$ adverse events while on pembrolizumab, she has experienced some minor grade 1 diarrhea, abdominal pain, and fatigue over her treatment course, but has not had any treatment interruptions.

\section{Discussion}

Primary melanocytic neoplasms of the pineal gland have been shown to be highly aggressive, and limited data exist to guide treatment decisions. Previously published case reports suggest that this patient population may be best served by a combination of gross total resection (GTR) followed by RT and systemic therapy. Unfortunately, GTR is often technically challenging given the proximity of the pineal region to the vein of Galen and other critical structures in the midbrain and thalamus. In these cases, a subtotal resection (STR) of tumor is the maximum safe resection. Of the 10 patients reported to have undergone attempted surgical resection, only 2 have successfully achieved a GTR. ${ }^{1-10}$ Due to incomplete survival data in previously published literature, it is not possible to infer the benefit of GTR over STR in these patients. Previously published case reports pertinent to this discussion can be found in Table $1 .^{1-17}$ Given the recent development of targeted and immunotherapeutic regimens, attempts at GTR should be tempered by maximal preservation of neurologic function. What does seem to be apparent is
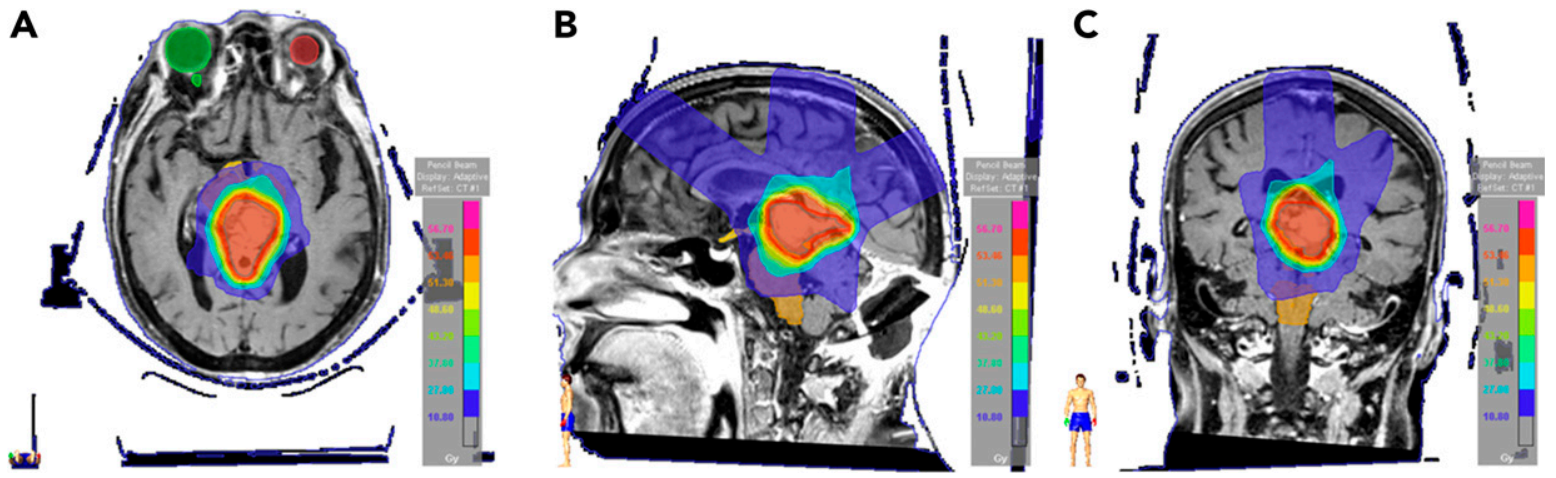

Figure 2. Radiation treatment plan to the residual pineal tumor after subtotal resection showing (A) axial, $(B)$ midplane sagittal, and ( $C)$ coronal views. The planning target volume included areas of gross residual disease and postoperative cavity expanded by 2 mm circumferentially. Radiation prescription was 54 Gy in 30 daily fractions of 1.8 Gy. Radiation was administered with a 5-field intensity-modulated radiation therapy technique. Image guidance was achieved with daily kilovoltage $x$-ray imaging using the ExacTrac system by Brainlab. 


\section{Table 1. Summary of All Published Cases of Primary Pineal Melanoma With Significant Treatment and Follow-Up Data (1977 to Current)}

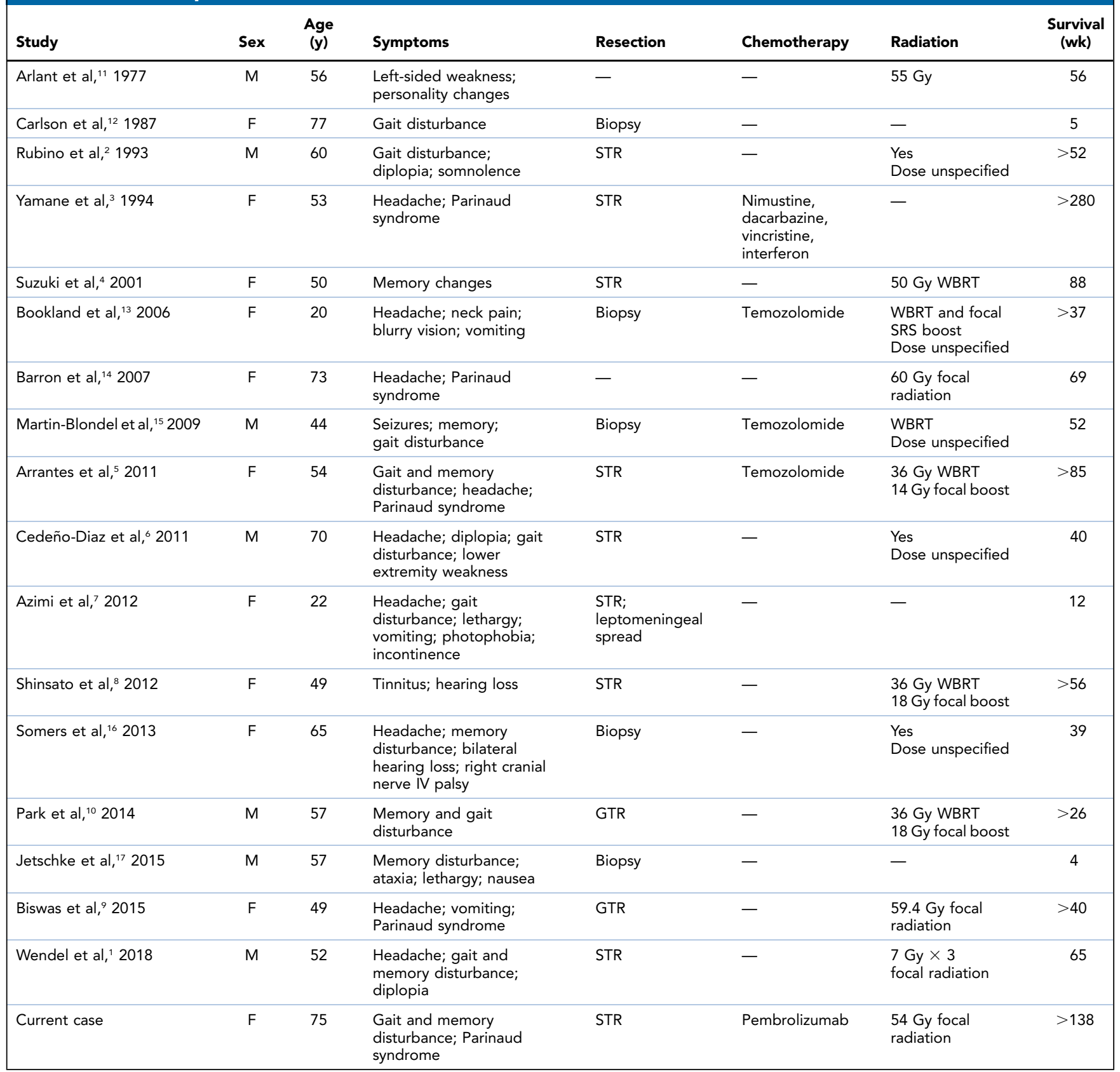

Case reports without reported treatment and survival data, and those published before 1977 have been omitted for concision; these are discussed in Cedeño-Diaz et al. ${ }^{6}$

Abbreviations: GTR, gross total resection; SRS, stereotactic radiosurgery; STR, subtotal resection; WBRT, whole-brain radiation therapy.

that STR without any adjuvant therapy is associated with a poor prognosis. There have been 16 cases presented in the literature that have undergone STR or biopsy, with survival follow-up data also reported..$^{1-10,12-16}$ Among the 3 patients with STR who did not receive adjuvant therapy, the reported OS were 4, 5, and 12 weeks., ${ }^{7,17}$ Given this dismal prognosis, it seems prudent to strongly recommend adjuvant therapy after resection, particularly in the event of an STR.

Adjuvant RT has been historically included as a mainstay of therapy for pineal melanoma, but the utility of whole-brain RT (WBRT) versus localized RT remains unknown. There have been 13 cases reported in the literature to have undergone adjuvant treatment after any surgical 
resection, and all but 1 have received radiation in some form. ${ }^{1-6,8-10,13-15,16}$ Radiation techniques in these series include WBRT, conventionally fractionated RT, SRS, or some combination of these. The data are not sufficiently robust to analyze differences between RT techniques. There have been 3 reported patients with leptomeningeal dissemination of disease, leading some oncologists to suggest a role for WBRT in an attempt to minimize this risk. ${ }^{7}$ Interestingly, these 3 patients were identified with leptomeningeal disease at presentation, and no other patient who presented with locally confined disease has been reported to experience recurrence with leptomeningeal dissemination despite many uses of localized RT techniques alone. The natural history of pineal melanoma clearly requires further study to appropriately comment on the utility of WBRT. We opted to administer locally directed RT to the pineal region in our patient, and have not seen leptomeningeal recurrence $>32$ months after completion of the radiation course.

The reported literature has been unclear regarding which systemic therapy is optimal in the adjuvant setting for pineal melanoma. Yamane et $\mathrm{al}^{3}$ reported mixed results with a combination of dacarbazine, nimustine, vincristine, and interferon. After undergoing a subtotal resection, their patient received adjuvant chemotherapy with 3 cycles of dacarbazine, vincristine, and nimustine, but experienced disease progression at 6 months within the surgical site. ${ }^{3}$ After recurrence, the patient was rechallenged with dacarbazine, nimustine, and vincristine with the addition of interferon, and achieved a clinical complete response for $>4$ years. There have been 3 other reported uses of systemic therapy, all implementing temozolomide, with mixed efficacy. ${ }^{4,5,13}$ The best response was described by Arantes et $\mathrm{al},{ }^{5}$ who reported on a patient with $>85$ weeks survival after treatment with combination temozolomide and RT following subtotal resection.

In our patient, we used molecular profiling (immunohistochemistry and next-generation sequencing) as a genetic screening tool, given her family history of male breast cancer and renal cell carcinoma, in addition to identifying any targetable mutations that could inform a systemic therapy decision. The patient's genomic profile was largely negative for targetable mutations, whereas immunohistochemistry showed strongly positive PD-L1 expression of $2+(70 \%)$. Given this insight, we were able to target PD-L1 with pembrolizumab, thus adding a new systemic therapy option to the literature, with excellent results providing proof of concept.

In addition to PD-L1, the patient's tumor profile identified an interesting pathogenic somatic mutation in $B A P 1$. Somatic BAP1 (BRCA1-associated protein) mutations have previously been identified as driver mutations in the pathogenesis of ocular melanomas, with roughly $40 \%$ of ocular melanoma carrying this driver mutation. ${ }^{18,19}$ Furthermore, somatic BAPl mutations have been associated with more aggressive tumors and increased metastatic events in ocular melanomas. ${ }^{20} \mathrm{Al}-$ though BAP1 mutations are rather prevalent and pathogenic in ocular melanoma, they are exceedingly rare in cutaneous melanomas and appear to be incompatible with cutaneous melanoma cell survival. ${ }^{18,19}$ Given these findings, it is possible that the presence of a somatic $B A P 1$ mutation in our patient could effectively rule out a melanoma metastasis from a cutaneous primary and support the diagnosis of a primary pineal tumor given the common embryologic tissue development between the eye and the pineal gland. Although the incidence and implication of $B A P 1$ mutations in primary pineal melanoma is unknown, it is an exciting marker for future studies and management strategies.

\section{Conclusions}

This is the first report of successful treatment of pineal melanoma using adjuvant fractionated RT and pembrolizumab immunotherapy, a treatment option suggested by next-generation genome sequencing analysis. Furthermore, this patient achieved the second longest OS reported using this combination $(>138$ weeks from diagnosis) and the longest reported progression-free survival. Although it is difficult to define the optimal management of primary pineal melanoma, it seems that this treatment strategy of maximal safe resection followed by focal radiation and adjuvant immunotherapy is both safe and effective.

\footnotetext{
Submitted March 25, 2019; accepted for publication June 25, 2019.
}

Disclosures: The authors have disclosed that they have not received any financial considerations from any person or organization to support the preparation, analysis, results, or discussion of this article.

Correspondence: Justin Famoso, MD, Department of Radiation Oncology, The University of Arizona, 3838 North Campbell Avenue, Building 2, Tucson, AZ 85719. Email: justin.famoso@arizonaccc.com

\section{References}

1. Wendel C, Kaech DL, Woodtli M. Primary malignant melanoma in the pineal region: case report and literature review. J Neurol Surg A Cent Eur Neurosurg 2018;79:344-352.

2. Rubino GJ, King WA, Quinn B, et al. Primary pineal melanoma: case report. Neurosurgery 1993;33:511-515; discussion 515

3. Yamane K, Shima T, Okada Y, et al. Primary pineal melanoma with long-term survival: case report. Surg Neurol 1994;42:433-437.

4. Suzuki T, Yasumoto Y, Kumami K, et al. Primary pineal melanocytic tumor. Case report. J Neurosurg 2001;94:523-527.

5. Arantes $\mathrm{M}$, Castro AF, Romão $\mathrm{H}$, et al. Primary pineal malignant melanoma: case report and literature review. Clin Neurol Neurosurg 2011;113:59-64.

6. Cedeño Diaz OM, Leal RG, La Cruz Pelea C. Primary pineal malignant melanoma. Clin Pract 2011;1:e31. 
7. Azimi P, Mohmmadi HR, Refiezadeh M. Primary pineal melanoma presenting with leptomeningeal spreading in a 22-year-old woman: a case report. J Med Case Reports 2012;6:165.

8. Shinsato $Y$, Hanada T, Kisanuki T, et al. Primary malignant melanoma in the pineal region treated without chemotherapy. Surg Neurol Int 2012;3:123.

9. Biswas A, Chaudhari PB, M SK, et al. Primary pineal malignant melanoma illustrated review. Turk Neurosurg 2015;25:201-209.

10. Park JH, Hong YK. Primary malignant melanoma in the pineal region. J Korean Neurosurg Soc 2014;56:504-508.

11. Arlant PA, Grunnet ML, Heilbrun MP. Primary malignant melanoma of the pineal region. Surg Neurol 1977;7:121-123.

12. Carlson $B R$, Glick $A D$, Cushman AR. Primary malignant melanoma of pineal region. J Tenn Med Assoc 1987;80:597-599.

13. Bookland M, Anderson WS, Biser-Rohrbaugh A, et al. Primary pinea malignant melanoma. Pediatr Neurosurg 2007;43:303-308.

14. Barron J, Morris-Larkin C, Finch $T$, et al. Long survival of primary pineal melanoma with radiation treatment only. Can J Neurol Sci 2007;34:251-253.
15. Martin-Blondel G, Rousseau A, Boch AL, et al. Primary pineal melanoma with leptomeningeal spreading: case report and review of the literature. Clin Neuropathol 2009;28:387-394.

16. Somers KE, Almast J, Biemiller RA, et al. Diagnosis of primary CNS melanoma with neuroimaging. J Clin Oncol 2013;31:e9-11.

17. Jetschke $K$, Viehweger $H$, Freesmeyer $M$, et al. Primary pineal malignant melanoma with B-Raf V600E mutation: a case report and brief review of the literature. Acta Neurochir (Wien) 2015; 157:1267-1270.

18. Masoomian B, Shields JA, Shields CL. Overview of BAP1 cancer predisposition syndrome and the relationship to uveal melanoma. J Curr Ophthalmol 2018;30:102-109.

19. Murali R, Wiesner T, Scolyer RA. Tumours associated with BAP1 mutations. Pathology 2013;45:116-126.

20. Harbour JW, Onken MD, Roberson EDO, et al. Frequent mutation of BAP1 in metastasizing uveal melanomas. Science 2010;330 1410-1413.

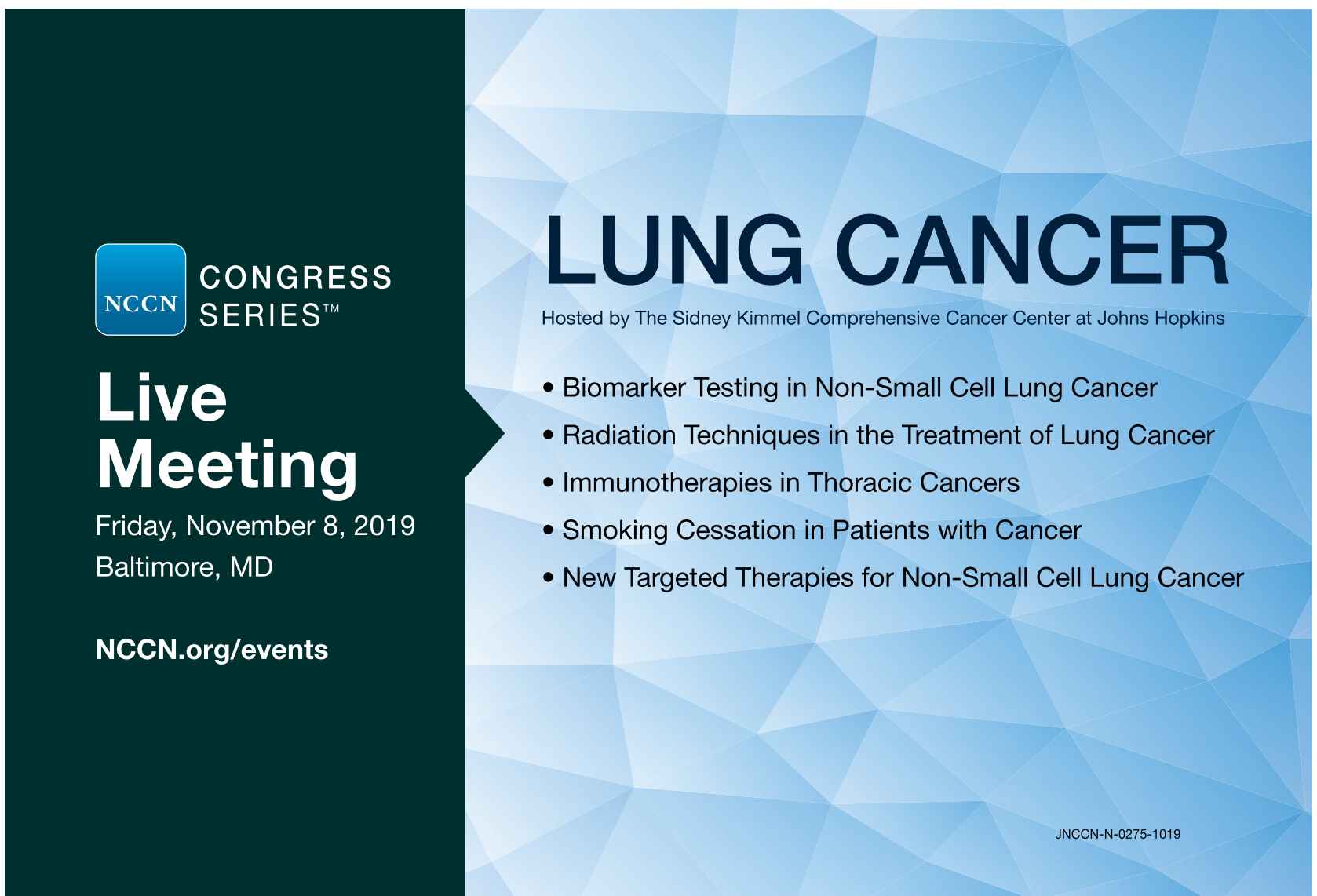

